\title{
Early Drop and Wavelength Grouping Schemes for Providing Absolute QoS Differentiation in Optical Burst-Switched Networks
}

\author{
Qiong Zhang, Vinod M. Vokkarane, Biao Chen, and Jason P. Jue \\ Dept. of Computer Science, The University of Texas at Dallas, Richardson, TX 75083-0688 \\ \{qzhang77, vinod, bchen, jjue\}@utdallas.edu
}

\begin{abstract}
A number of schemes have been proposed to support QoS in optical burst-switched (OBS) networks. Most schemes only support relative QoS differentiation instead of absolute QoS guarantee. However, absolute QoS differentiation is a basic requirement for many delay and loss sensitive applications. In this paper, we propose two mechanisms for providing absolute QoS differentiation in OBS networks, an early drop mechanism, which selectively drops non-guaranteed traffic, and a wavelength grouping mechanism, which manages wavelengths for guaranteed traffic. We show that the combination of these two mechanisms not only outperforms other schemes in providing loss guarantees, but also improves the loss performance of nonguaranteed traffic.
\end{abstract}

\section{INTRODUCTION}

The explosive growth of the Internet demands a highspeed transmission technology to support rapidly increasing bandwidth requirements. Currently, DWDM technology achieves multiplexing of 160-320 wavelengths in one fiber, with 10-40 Gb/s transmission rate per wavelength. In order to efficiently utilize the raw bandwidth in DWDM networks, an all-optical transport method, which supports fast resource provisioning and asynchronous transmission of variable-sized packets, must be developed. Optical burst switching (OBS) is a promising bufferless DWDM switching technology that can provide high wavelength utilization. OBS employs a signaling scheme in which an out-of-band burst header packet (BHP) is first sent to reserve resources along the path of the data burst. After an offset time, the data burst is transmitted all-optically through the network. One example of such a signaling scheme is just-enough-time (JET) [1].

Several scheduling algorithms have been proposed for burst scheduling at an OBS node. One simple and practical algorithm is the latest available unscheduled channel (LAUC) algorithm [2]. In LAUC, each arriving burst is scheduled on the latest available channel, thereby minimizing unused gaps between the scheduled bursts on channels. If the node cannot find an available outgoing channel, the burst will be dropped upon its arrival.

QoS support is an important issue in OBS networks. There are two models for QoS: relative and absolute QoS. In the relative QoS model, the performance of each class is not defined quantitatively in absolute terms based on loss or delay QoS metrics. Instead, the QoS of one class is defined relatively in comparison to other classes. For example, a class of high priority is guaranteed to experience lower loss probability than a class of lower priority. However, the loss probability of a high-priority class still depends on the traffic load of other classes; and no upper bound on the loss probability is guaranteed for the high-priority class. Several schemes have been proposed to support the relative QoS model in OBS networks. An offset-based scheme that provides relative loss differentiation was proposed in [3]. In [4], a proportional QoS scheme based on per-hop information was proposed to support burst loss probability and delay differentiation. Also, in [5], proportional differentiation is provided by preempting scheduled bursts, in order to maintain the number of wavelengths occupied by each class of burst.

The absolute QoS model aims to provide a worst-case guarantee on loss and delay to applications. This kind of hard guarantee is essential for many delay and loss sensitive applications, which include multimedia applications and mission-critical applications. Efficient admission control and resource provisioning mechanisms are needed to support the absolute QoS model. Due to the bufferless nature of OBS, the critical aspect of supporting absolute QoS in OBS networks is to address the loss guarantee, since delay primarily consists of the propagation delay. To the best of our knowledge, there is no scheme in the literature to support worst-case loss guarantees, and hence absolute QoS in OBS networks.

The primary objective of this paper is to develop a scheme that is able to support the worst-case loss guarantee for various traffic classes. We propose two mechanisms to achieve the goal, namely, early drop and wavelength grouping. The integration of these two mechanisms provides a very efficient solution to offer worst-case loss guarantees to certain classes of traffic and, at the same time, to dramatically improve the loss performance of non-guaranteed traffic. Our scheme assumes that the signaling protocol is JET and the burst scheduling algorithm is LAUC.

The paper is organized as follows. Section II describes the network architecture to support the end-to-end absolute QoS model. Section III and Section IV introduce the early drop and wavelength grouping mechanisms. Section V discusses the integration of early drop and wavelength grouping. The analytical model for the integrated early drop and dynamic wavelength grouping scheme is developed in Section VI. Section VII studies the performance of the proposed scheme based on the analytical model as well as simulation. Section VIII briefly discusses the ongoing and future work. 
Fig. 1. OBS transport network.

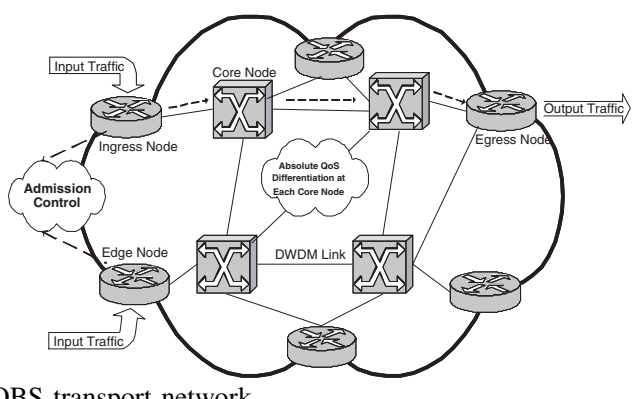

II. Network ARChitecture

This section describes the architecture of a typical OBS network. An OBS transport network consists of a collection of edge and core nodes as shown in Fig. 1. The traffic from multiple client networks is assembled at the ingress edge nodes and transmitted as bursts through high capacity DWDM links over the core. The egress edge nodes, upon receiving a burst, deliver the data to the corresponding client networks. Absolute QoS guarantee relies on proper resource provisioning and admission control. Each service class $i$ is assumed to require an end-to-end loss guarantee, $P_{C_{i}}^{N E T}$. Given that each OBS node maintains the same loss guarantee $P_{C_{i}}^{M A X}$ for Class $i$ traffic, we can estimate the $P_{C_{i}}^{M A X}$ at each node from the diameter of the network, $D$, and $P_{C_{i}}^{N E T}$ as follows:

$$
P_{C_{i}}^{M A X}=1-e^{\left(\ln \left(1-P_{C_{i}}^{N E T}\right)\right) / D} .
$$

Therefore, if $P_{C_{i}}^{M A X}$ is guaranteed at each node along the path, the end-to-end loss of Class $i$ traffic $\left(P_{C_{i}}^{N E T}\right)$ is guaranteed.

As we have discussed in the previous section, since core nodes do not have any buffers and bursts follow an alloptical path from source to destination, the delay is mainly propagation delay. Hence, in this paper, we focus on the issue of how to achieve worst-case loss guarantee in OBS networks.

Admission control is typically implemented at edge nodes because the edge nodes have electronic buffering capability. The traffic arrival rate between a source-destination pair can also be monitored and controlled to be under a maximum allowed arrival rate at the edge node during burst assembly. Based on the maximum arrival rate of the guaranteed traffic and the network topology, we can obtain the maximum offered load of the guaranteed traffic on every link. For each link $l$, let $L_{C_{i}}$ be the maximum offered load of Class $i$ traffic, and let $W_{C_{i}}$ be the number of wavelengths required in order to guarantee that the loss probability of Class $i$ traffic is below $P_{C_{i}}^{M A X}$. We can compute $W_{C_{i}}$ for the guaranteed traffic of Class $i$ using the standard Erlang-B equation:

$$
\frac{\frac{L_{C_{i}}^{W_{C_{i}}}}{W_{C_{i}} !}}{\sum_{x=0}^{W_{C_{i}}} \frac{L_{C_{i}}^{x}}{x !}} \leq P_{C_{i}}^{M A X} .
$$

Hence, in order to guarantee the maximum end-to-end loss, each core node must provide at least $W_{C_{i}}$ wavelengths and must guarantee the maximum per-hop loss, $P_{C_{i}}^{M A X}$, for each Class $i$ traffic.

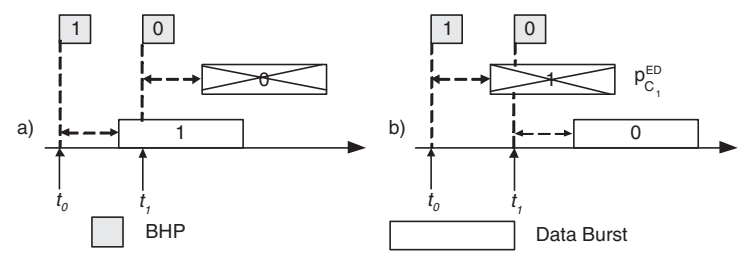

Fig. 2. (a) Standard Drop Mechanism, and (b) Early Drop Mechanism.

\section{EARLY DROP MEChanism}

In this section, we describe the early drop mechanism to guarantee the worst-case loss probability of Class $i$ traffic, $P_{C_{i}}^{M A X}$, at each node. The early drop mechanism is a probability-based technique in which the bursts of a less important class (say Class $j$ ), are intentionally dropped with some probability (say $p_{C_{j}}^{E D}$ ) to avoid contention with bursts of a more important class (say Class $i$ with $i<j$ ). In Fig. 2(a), the BHP of a Class 1 burst (low priority) arrives at time $t_{0}$ and reserves the wavelength. The BHP of a Class 0 burst (high priority) arrives at time $t_{1}$, where $t_{0}<t_{1}$, and contends with the Class 1 burst, resulting in the Class 0 burst being dropped. In order to reduce the likelihood of this scenario, the early drop mechanism drops the Class 1 burst with probability $p_{C_{1}}^{E D}$ prior to the BHP arrival of the Class 0 burst, as shown in Fig. 2(b). $p_{C_{1}}^{E D}$ is a function of the maximum acceptable loss probability of Class 0 bursts and the on-line measured loss probability of Class 0 bursts. The key is to decide when to trigger the early drop mechanism, and how to set the early dropping probability, $p_{C_{1}}^{E D}$.

In order to guarantee the loss, each OBS core node monitors the traffic statistics for each guaranteed class. For each output port of an OBS node, let $a_{C_{i}}$ be the burst arrival counter and $d_{C_{i}}$ be the burst drop counter for Class $i$ traffic. We use $p_{C_{i}}=$ $\left(d_{C_{i}} / a_{C_{i}}\right)$ as the on-line burst loss probability for Class $i$ bursts. For this purpose, $a_{C_{i}}$ and $d_{C_{i}}$ can be measured within a fixed time window. We propose the following early-dropby-span (EDS) scheme to support loss guarantee.

Without loss of generality, we describe the early drop mechanism using a two-class network. Here, a span of acceptable loss probabilities, $\delta_{C_{0}}$, for Class 0 is chosen. The EDS scheme is triggered when the on-line measured loss probability of Class 0 bursts, $p_{C_{0}}$, is higher than $P_{C_{0}}^{M I N}$, where, $P_{C_{0}}^{M I N}=P_{C_{0}}^{M A X}-\delta_{C_{0}}$. The Class 1 bursts are dropped with a probability $p_{C_{1}}^{E D}$ given by:

$$
p_{C_{1}}^{E D}= \begin{cases}0 & p_{C_{0}}<P_{C_{0}}^{M I N} \\ \left(p_{C_{0}}-P_{C_{0}}^{M I N}\right) / \delta_{C_{0}} & P_{C_{0}}^{M I N} \leq p_{C_{0}}<P_{C_{0}}^{M A X} \\ 1 & p_{C_{0}} \geq P_{C_{0}}^{M A X}\end{cases}
$$

The span $\left(\delta_{C_{0}}\right)$ can be chosen as a percentage value of $P_{C_{0}}^{M A X}$. We observe that, if $\delta_{C_{0}}$ is high, EDS is triggered prematurely, leading to high loss of Class 1 traffic; while, if $\delta_{C_{0}}$ is low, $p_{C_{1}}^{E D}$ will be high, also resulting in high loss of Class 1 traffic.

The EDS scheme can be extended to support multiple classes with guaranteed loss. In this case, the early dropping probability of each class is dependent on the maximum loss probability and the span of each higher-priority class. 


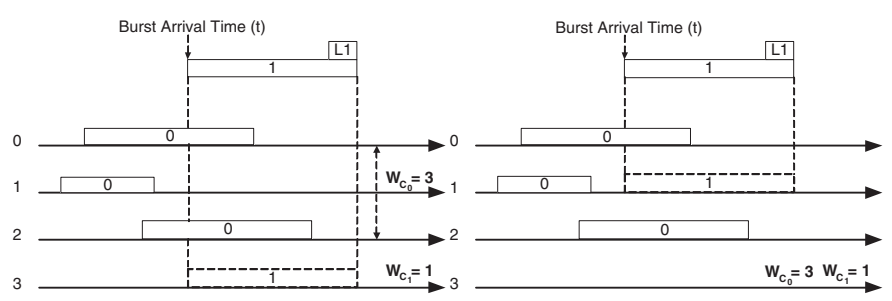

Fig. 3. Illustration of (a) SWG, and (b) DWG schemes.

\section{Wavelength Grouping Mechanism}

This section describes the wavelength grouping mechanism for providing absolute loss differentiation at each OBS node. The wavelength grouping mechanism classifies the traffic into groups, each of these groups is assigned a unique label and can use a provisioned set of wavelengths. One way to classify the traffic into groups is to assign the traffic with the same service class into a group. On each link $l$, we obtain $W_{C_{i}}$ and $P_{C_{i}}^{M A X}$ for each guaranteed Class $i$ traffic and assign the Class $i$ traffic a local label $L i$. Link $l$ must provide at least $W_{C_{i}}$ wavelengths for bursts with Label $L i$. If we run out of wavelengths, then the requirement of the guaranteed traffic cannot be satisfied with the given network capacity. We propose two schemes for provisioning resource, namely, static wavelength grouping and dynamic wavelength grouping. Let us consider a two-class network, where Class 0 burst is assigned Label $L 0$, and Class 1 burst is assigned Label $L 1$.

\section{A. Static Wavelength Grouping (SWG)}

In SWG, for the traffic with a given label, a fixed set of wavelengths is dedicated. If $W_{C_{0}}$ wavelengths on link $l$ are necessary to support bursts with Label $L 0$, the first $W_{C_{0}}$ wavelengths $\left(w_{0}, w_{1}, \ldots, w_{\left(W_{C_{0}}-1\right)}\right)$ are reserved for bursts with Label $L 0$. Furthermore, Label $L 0$ bursts can only use these $w_{0}, w_{1}, \ldots, w_{\left(W_{C_{0}}-1\right)}$ wavelengths on the link $l$. In the case of guaranteeing more than one class of traffic, the process is repeated until the necessary wavelengths have been reserved for all of the guaranteed traffic. The remaining unreserved wavelengths are used to carry the best-effort traffic. Let us consider the scenario shown in Fig. 3(a). When a Label L1 burst arrives at time $t$, it can only be scheduled on Wavelength 3 , which is statically preassigned to the bursts with Label $L 1$.

\section{B. Dynamic Wavelength Grouping (DWG)}

In DWG, for the traffic with a given label, a fixed number of wavelengths is reserved, albeit not a fixed set of wavelengths. To ensure that the number of wavelengths occupied by bursts with a given label does not exceed the provisioned number, the OBS node must keep track of the number of wavelengths occupied by bursts of each given label. A burst can be dynamically scheduled onto an available wavelength if the number of wavelengths occupied by bursts of its label is still less than the provisioned number of wavelengths. In Fig. 3(b), the maximum number of wavelengths that Label $L 1$ bursts can use is one. When a Label $L 1$ burst arrives at time $t$,

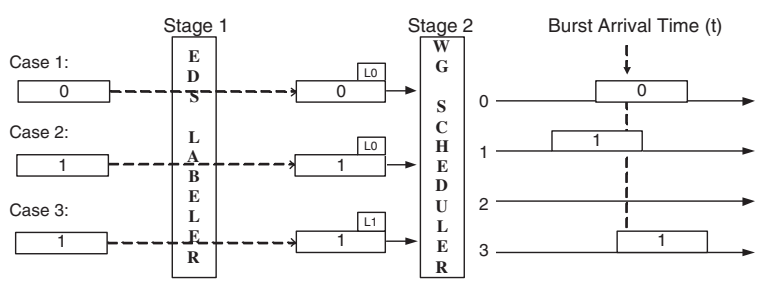

Fig. 4. Illustration of the integrated schemes.

Wavelength 1 and Wavelength 3 are available and no Label $L 1$ bursts are already scheduled. Hence, the arriving burst is scheduled on Wavelength 1 , which has the latest available unscheduled time.

Comparing SWG and DWG, we note that SWG is less complex and simpler to implement. However, DWG has the advantage of being able to dynamically schedule a burst onto a best wavelength, based on the link status, in order to optimize network performance.

\section{INTEGRATED SCHEMES}

Under wavelength grouping, Class 1 traffic cannot use the wavelengths assigned to Class 0 traffic, even when the loss probability of Class 0 traffic is much lower than its guaranteed loss probability. This restriction results in bandwidth waste and may hurt the utilization of the OBS network. On the other hand, EDS monitors the loss probability of Class 0 traffic and keeps it below the guaranteed loss probability by probabilistically dropping Class 1 bursts. The EDS scheme can cause excessive dropping of Class 1 bursts because EDS does not consider the maximum arrival rate of Class 0 bursts. The two mechanisms can complement with each other. Therefore, to achieve better performance, we integrate EDS with wavelength grouping.

In the integrated schemes, we use EDS as the primary scheme, and schedule the bursts using wavelength grouping. In the following discussion, we use the same two-class example. First, EDS labels each burst according to the class of the burst and the current loss probability of Class 0 traffic. A burst is assigned a local label $L 0$, either if the burst is of Class 0 , or if the burst is of Class 1 but is not probabilistically dropped by EDS. A burst is assigned a local label $L 1$ if the burst should be dropped according to EDS. The labeled burst is then sent to the WG scheduler, which schedules the burst solely based on its label. All the bursts labeled $L 0$ will be scheduled using anyone of the available wavelengths according to LAUC. All the bursts labeled $L 1$ can only be scheduled on maximum $W_{C_{1}}$ wavelengths, where $W_{C_{1}}=W-W_{C_{0}}$, in order to satisfy the minimum number of wavelengths required for Label $L 0$ traffic.

\section{A. Integrated EDS and $S W G$}

Using SWG, we statically assign $W_{C_{1}}$ wavelengths to bursts with Label $L 1$. In order to guarantee the maximum loss of Class 0 traffic, a burst with Label $L 0$ can be scheduled on any wavelength, while a burst with Label $L 1$ can only be 
scheduled on the preassigned $W_{C_{1}}$ wavelengths. In Fig. 4 we illustrate three possible burst arrival scenarios. The current wavelength allocation is shown on the right hand side of the figure. In Case 1, when a Class 0 burst with Label $L 0$ arrives at time $t$, the burst is scheduled on Wavelength 2. In Case 2, a Class 1 burst with Label $L 0$ arriving at time $t$, can also be scheduled on Wavelength 2 . While in Case 3, a Class 1 burst with Label $L 1$ arriving at time $t$, cannot be scheduled on Wavelength 2 , since a Label $L 1$ burst can be scheduled only on the statically provisioned Wavelength 3 .

\section{B. Integrated EDS and DWG}

Following LAUC, the DWG scheduler records the label of the latest scheduled burst on every wavelength. In the case of a burst arrival with Label $L 0$, DWG can schedule the burst on any available wavelength. On the other hand, when a burst with Label $L 1$ arrives, the burst is scheduled on any of the available wavelengths, as long as the number of Label $L 1$ bursts scheduled at the arrival time of the burst is less than $W_{C_{1}}$. In Fig. 4, the label of the latest scheduled burst recorded on each of Wavelengths 0,1 , and 3 is $L 0$. Thus, with the DWG scheduler, for all three burst arrival scenarios, the arriving burst can be scheduled on Wavelength 2 .

The integrated schemes perform better resource allocation as compared to the stand-alone schemes for the following reasons: in the wavelength grouping schemes, Class 1 bursts can be scheduled only on $W_{C_{1}}$ wavelengths, while, in the integrated schemes, Class 1 bursts with Label $L 0$ can be scheduled on any wavelength; in the integrated schemes, the unnecessary intentional dropping of Class 1 bursts is avoided, since the Class 1 bursts with Label $L 1$ can use a maximum of $W_{C_{1}}$ wavelengths.

\section{Analytical Model}

In this section, we develop an analytical model for the Integrated EDS and DWG scheme. Again, we use the same example of a two-class network. Let $W$ be the total number of wavelengths on each link, and let $W_{C_{0}}$ be the minimum number of wavelengths provisioned for the Class 0 traffic. The Class 1 traffic that is decided to be dropped by EDS can be scheduled on $W_{C_{1}}=W-W_{C_{0}}$ wavelengths. In the model, we assume that the total burst arrival to a node is Poisson with rate $\lambda$. The bursts of Class 0 have an arrival rate of $\lambda_{C_{0}}$, and the bursts of Class 1 have an arrival rate of $\lambda_{C_{1}}$. In EDS, the Class 1 bursts are randomly dropped with probability $p_{C_{1}}^{E D}$. Let $P_{C_{1}}^{E D}$ be the mean of $p_{C_{1}}^{E D}$ at steady state. Let $\lambda_{L 0}$ be the portion of traffic that is labeled $L 0$ by EDS and can be scheduled on $W$ wavelengths; let $\lambda_{L 1}$ be the remaining traffic that is labeled $L 1$ by EDS and can scheduled on the $W_{C_{1}}$ wavelengths. Therefore, the effective arrival rate of the Label $L 0$ traffic is:

$$
\lambda_{L 0}=\lambda_{C_{0}}+\left(1-P_{C_{1}}^{E D}\right) \lambda_{C_{1}},
$$

and the effective arrival rate of the Label $L 1$ traffic is:

$$
\lambda_{L 1}=P_{C_{1}}^{E D} \lambda_{C_{1}} .
$$

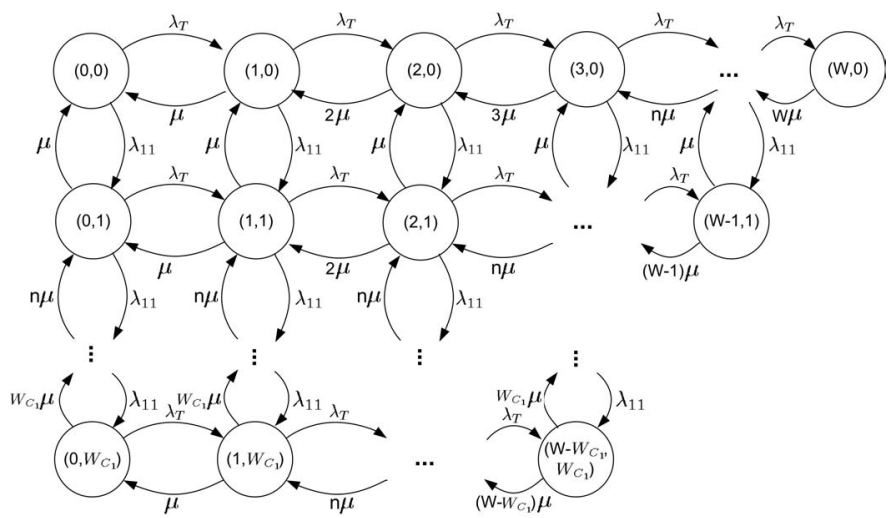

Fig. 5. Markov chain for Integrated EDS and DWG scheme.

Based on the property of a Poisson process, the arrival processes of Label $L 1$ and Label $L 0$ traffic are also Poisson processes. We assume that the burst length is exponentially distributed with an average burst length of $1 / \mu$. The span of EDS is $\delta_{C_{0}}$.

Since all the bursts are delayed by the same offset time at edge nodes according to JET, we model each outgoing link as a continuous time Markov chain with the state defined as $X=\left\{x_{0}, x_{1}\right\}$, where $x_{0}$ is the number of wavelengths that are busy serving $L 0$ bursts and $x_{1}$ is the number of wavelengths that are busy serving $L 1$ bursts. The state transition diagram for the Markov chain is shown in Fig. 5 and the state transition rates are as follows:

$$
\begin{gathered}
q_{\left(x_{0}, x_{1}\right)\left(x_{0}, x_{1}+1\right)}= \begin{cases}\lambda_{L 1} & x_{0}+x_{1}<W \& x_{1}<W_{1} \\
0 & \text { otherwise },\end{cases} \\
q_{\left(x_{0}, x_{1}\right)\left(x_{0}+1, x_{1}\right)}= \begin{cases}\lambda_{L 0} & x_{0}+x_{1}<W \\
0 & \text { otherwise }\end{cases} \\
q_{\left(x_{0}, x_{1}\right)\left(x_{0}, x_{1}-1\right)}= \begin{cases}x_{1} \mu & x_{1}>0 \\
0 & \text { otherwise }\end{cases} \\
q_{\left(x_{0}, x_{1}\right)\left(x_{0}-1, x_{1}\right)}= \begin{cases}x_{0} \mu & x_{0}>0 \\
0 & \text { otherwise. }\end{cases}
\end{gathered}
$$

From the Markov chain, we can solve the steady-state probabilities $p\left(x_{0}, x_{1}\right)$. The probability that a burst labeled $L 0$ is dropped by the DWG scheduler is equal to the probability that all of the wavelengths are busy. Thus, the loss probability for labeled $L 0$ traffic is given by:

$$
P_{L 0}=\sum_{i=0}^{W_{C_{1}}} p(W-i, i) .
$$

A Label $L 1$ burst is dropped either when all wavelengths are occupied, or when the number of wavelengths occupied by Label $L 1$ bursts is $W_{C 1}$, but all of the wavelengths are not fully occupied. Therefore, the loss probability for labeled $L 1$ traffic is as follows,

$$
P_{L 1}=P_{0}+\sum_{i=0}^{W-W_{C_{1}}-1} p\left(i, W_{C_{1}}\right) .
$$



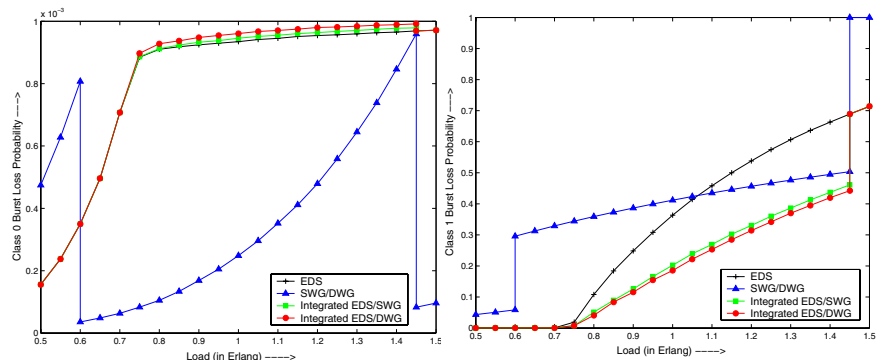

Fig. 6. (a) Class 0 , and (b) Class 1 loss probability versus load for the proposed absolute QoS schemes.

Hence, the loss probabilities of Class 0 and Class 1 bursts are given as,

$$
\begin{gathered}
P_{C_{0}}=P_{L 0}, \\
P_{C_{1}}=P_{L 1} P_{C_{1}}^{E D}+P_{C_{0}}\left(1-P_{C_{1}}^{E D}\right),
\end{gathered}
$$

where $P_{C_{1}}^{E D}$ is calculated from $P_{C_{0}}$ using (3).

\section{NUMERICAL RESULTS}

A simulation is developed to evaluate the performance of the proposed schemes and to verify the analytical model. We use the same two-class network example, with Class 0 (high) and Class 1 (low) bursts. Burst arrivals follow a Poisson process. Burst lengths are exponentially distributed with an average burst length of $100 \mu \mathrm{s}$. The number of wavelengths on each link is 5 . The transmission rate on a wavelength is $10 \mathrm{~Gb} / \mathrm{s}$. There is no buffering in the core. The absolute loss guarantee for Class 0 traffic is $P_{C_{0}}^{M A X}=0.001$. The traffic of Class 1 receives best-effort service. $30 \%$ of the traffic is Class 0 and $70 \%$ of the traffic is Class 1 . We set the span of the EDS scheme, $\delta_{C_{0}}=0.1 P_{C_{0}}^{M A X}$.

Figs. 6(a) and (b) show the relationships between burst loss probability and traffic load under four proposed schemes for Class 0 and Class 1 respectively. In the absence of EDS, the loss performance of SWG and DWG are almost identical since we use LAUC to select the latest available wavelength. We observe from Fig. 6(a) that all four schemes can meet the loss requirement of Class 0 bursts. However, they differ in their support of Class 1 traffic as illustrated in Fig. 6(b).

At loads below 0.6 Erlang, both SWG and DWG only use three wavelengths for Class 0 , while the other three schemes can use all five wavelengths, resulting in lower loss probability for Class 0 traffic. As shown from Fig. 6(a), between 0.6 Erlang and 1.45 Erlang, SWG/DWG has the lowest loss probability for Class 0 . This is because more wavelengths are dedicated to the Class 0 traffic. However, over-allocation of wavelengths by SWG/DWG can cost wavelength utilization, which leads to less throughput for Class 1 traffic, as in the case when the load is above 1.45 Erlang. When load is higher than 1.45 Erlang, the minimum number of wavelengths required for Class 0 is five. Therefore, the SWG/DWG scheduler drops all of the Class 1 bursts, while the integrated schemes only drop all Label $L 1$ bursts. From Fig. 6(b), the two integrated schemes perform much better than SWG/DWG. Among the
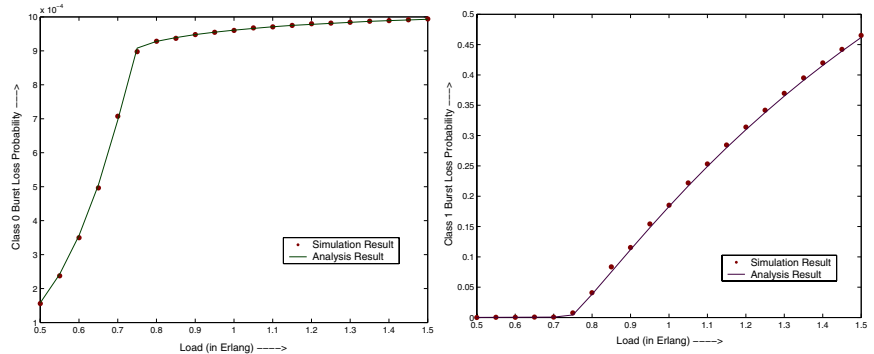

Fig. 7. (a) Class 0, and (b) Class 1 burst loss probability versus load using analytical model, and simulation results.

four schemes, the integrated EDS and DWG has the best performance because the Class 1 bursts have the flexibility of being assigned on any available wavelength. Also, $p_{C_{1}}^{E D}$ increases as the load increases in EDS, hence, the loss probability of Class 1 traffic increases. The turning points of curves in Fig. 6 at loads of 0.6 Erlang and 1.45 Erlang match the changes in the minimum number of wavelengths required to guarantee the loss probability of Class 0 traffic.

The results from the analytical model for the same network example are shown in Fig. 7(a) and (b). The parameters used in Fig. 7(a) and (b) are: $W=5$ and $W_{C_{1}}=1$. These parameters correspond to the system loads from 0.6 Erlang to 1.45 Erlang. The analytical results match very closely with simulation results.

\section{CONCLUSION}

In this paper, we addressed the issue of how to support absolute QoS in an OBS network. We developed two mechanisms, namely early drop and wavelength grouping, and integrated these two schemes to support loss guarantee in OBS networks. We have shown that the integrated early drop by span with dynamic wavelength grouping scheme has the best performance. We also established an analytical model for this integration scheme, which matches closely with the simulation results.

Areas of future work include developing a framework for admission control and resource provisioning at the edge OBS nodes, and developing schemes that will allow different maximum loss guarantee for source-destination pairs with different hop distances, but guarantee the same end-to-end loss probability.

\section{REFERENCES}

[1] C. Qiao and M. Yoo, "Optical Burst Switching (OBS) - A New Paradigm for an Optical Internet," Journal of High Speed Networks, vol. 8, no.1, pp. 69-84, Jan. 1999.

[2] J.S. Turner, "Terabit Burst Switching," Journal of High Speed Networks, vol. 8, no. 1, pp. 3-16, 1999.

[3] M. Yoo, et. al, "QoS Performance of Optical Burst Switching in IPOver-WDM Networks," IEEE Journal on Selected Areas in Communications, vol. 18, no. 10, pp. 2062-2071, Oct. 2000.

[4] Y. Chen, et. al, "Proportional QoS over OBS Network," Proceedings, IEEE, GLOBECOM 2001, vol. 3, pp. 1510-1514, San Antonio, TX, Nov. 2001.

[5] C.-H. Loi, W. Liao, and D.-N. Yang, "Service Differentiation in Optical Burst Switched Networks," Proceedings, IEEE Globecom 2002, vol. 3, pp. 2313-2317, Taipei, Taiwan, November 2002. 